Mater. Sci. Bull., Vol. 1, No. 1, May 1979, pp. 15-34, () Printed in India.

\title{
Alpha-quartz 1. Crystallography and crystal defects
}

\author{
PRASENJIT SAHA, N ANNAMALAI and TARUN \\ BANDYOPADHYAY \\ Central Glass and Ceramic Research Institute, Jadavpur, Calcutta 700032 \\ MS received 16 October 1978
}

\begin{abstract}
Crystallography of alpha-quartz is discussed with special reference to the existing ambiguities regarding handedness of its enantiomorphic forms and a mnemonic has been suggested. Previous $x$-ray diffraction topographic studies of synthetic quartz are critically reviewed and analysed to understand the origin, nature and location of dislocations. It is suggested that dislocations associated with cell boundaries, characteristic of the Z-zone grown portions of synthetic quartz, are pure a-type edge dislocations but possibly with an alternating non-conservative climb component associated with the predominating glide component.
\end{abstract}

\section{Introduction}

X-ray transmissicn topographic studies carried out by Lang and Miuscov (1967) revealed for the first time the presence of cellular structure in synthetic quartz; this feature characterises synthetic quartz grown on (0001)-cut surface of either natural or synthetic quartz seed rod or plate, from natural quartz which does not possess such a feature. Cellular growth was detected in the grown Z-zones, and it was correlated with the cobble topography of the terminal (0001) faces of synthetic quartz which do not constitute a natural morphological crystal form of quartz.

It has been possible to make certain postulates about the origin of cellular structure of synthetic quartz on the basis of hydrothermal dissclution experiments. The crystallography and the nature and distribution of dislocations in alpha-quartz single crystals, specially in synthetic quartz have been critically reviewed and analysed in this paper.

\section{Crystallography of quartz}

Quartz $\left(\mathrm{SiO}_{2}\right)$, occurs in two polymorphic forms, namely, low- or alpha-quartz and high- or beta-quartz. Alpha-quartz crystallises in the trigonal system and beta-quartz in the hexagonal system. On heating, alpha-quartz transforms to beta-quartz at $573^{\circ} \mathrm{C}$ with an increase in volume of about $1 \%$ (recalculated from 
specific volume change at $573^{\circ} \mathrm{C}$ given in figure 147 of Muan and Osborn 1965); on cooling, beta-quartz almost instantaneously reverts to alpha-quartz. This fast polymorphic transformation is of the displacive type involving mincr clianges in the second sphere of co-ordination (Kingery et al 1967). The accompanying consequent change in specific volume, however, causes shattering of the crystals.

Each polymorphic form of quartz exists in two enantiomorphic forms. Designation of the enantiomorphic forms has created some doubts, since the "hand", either left-handed or right-handed, can be defined in several ways, such as, nature of reference framework of the co-ordinate axes, orientation of the rhombohedral axes with respect to the hexagonal axes (Lang 1965), the morphological ccnfiguration and the absolute configuration (De Vries 1958) and so on. Lang (1965) had attempted to clear up these doubts by suggesting a useful mnemonic for the "correct, obverse orientation of the Miller-Bravais axes with respect to the a-quartz structure" to conform to the International Tables (Anon 1969).

The two enantiomorphs of alpha-quartz belong to space groups $\mathbf{P} 3_{1} 21$ and $\mathbf{P} 3_{2} 21$. Using data given by Wyckoff (1948) for $P 3_{1} 21$ and adapting them to International Tables (Anon 1969) for $\mathrm{P}_{2} 21$ by adding $2 / 3$ to the $z$-coordinates the following results are obtained for alpha-quartz ${ }^{*}$ :

\subsection{Low- or alpha-quartz}

Crystal system: trigonal; space groups of enantiomorphic forms: $\mathbf{P} 3_{1} 21, \mathbf{P 3}_{2} \mathbf{2 1}$; $a_{0}=4.903 \AA, c_{0}=5.393 \AA$ presumably at room temperature.

The P3 ${ }_{2} 21$ enantiomorph: $3 \mathrm{Si}$ in (a), $x_{\mathrm{st}}=0.465 ; 60$ in (c), $x_{0}=0.415, y_{0}=0.272$, $z_{0}=0 \cdot 79$.

Again, using Wyckoff's (1948) data for beta-quartz, it can be shown that the $P 3_{2} 21$ enantiomorph of alpha-quartz is compatible with the $\mathrm{P}_{2}, 22$ enantiomorph of beta-quartz with the origin shifted by $2 / 3$ along the positive direction of the $c$-axis, that is, the polymorphic transformation of the $\mathrm{P}_{2} 21$ enantiomorph of alpha-quartz should be to the $P 6_{2} 22$ enantiomorph of beta-quartz. The following results can therefore be obtained for beta-quartz:

\subsection{High- or beta-quartz}

Crystal system: hexagonal; space groups of enantiomorphic forms: $\mathbf{P} \mathbf{6}_{2} 22$, $\mathrm{P}_{4} 22 ; a_{0}=5.01 \AA, c_{0}=5.47 \AA$ at ca. $600^{\circ} \mathrm{C}$.

The $P_{2} 22$ enantiomorph: $3 \mathrm{Si}$ in (c); 60 in (j), $x_{0}=0 \cdot 197$. Lang's mnemonic is as follows : The origin is placed on one of the three-fold screw axes running parallel to the $c$-axis and intersecting the horizontal two-fold axes; this convention has been followed in the International Tables. In (0001) projection the six

\footnotetext{
- Wyckoff (1948) data for $\mathrm{P}_{1} 21$ cannot be fitted to the corresponding space group of International Tables (Anon 1969), since Wyckoff and later Frondel (1962), have used left-handed co-ordinate system (crystallographically left-handed spiral of single pitch) in right-handed axis for $\mathbf{P 3}_{\mathbf{2}} \mathbf{2 1}$, in contrast to Wyckoff (1931) whereas in the International Tables a right-handed co-ordinate system (crystallographically right-handed spiral of single pitch) is used in right-handed axes for $\mathbf{P 3}_{1}$ 21. However, their data for $\mathbf{P 3}_{1} 21$ can be fitted to International Tables, space group $\mathbf{P 3}_{2} \mathbf{2 1}$, by adding $2 / 3$ to Wyckoff $z$-coordinates only.
} 
silicon atoms would then form a distorted hexagon, the sides of which would alternately include angles more acute and more obtuse than $120^{\circ}$. Looking towards the origin, the $a_{1}, a_{2}$ and $a_{3}$ axes would run outwards from the more obtuse corners in the case of the $\mathrm{P}_{1} 21$ enantiomorph, and from the more acute corners in the case of the $\mathrm{P}_{2} 21$ enantiomorph. Figure 1a illustrates the structure of the $\mathbf{P 3}_{2} 21$ enantiomorph of alpha-quartz in (0001) projection*, as deduced from adaptation of Wyckoff data. It can be seen from figure 2 that Lang's mnemonic is obeyed.

Figure $1 \mathrm{~b}$ illustrates a (2î10) projection of the $\mathrm{P}_{2} 21$ enantiomorph. The $a_{1}$-axis is normal to the plane of projection. The dashed rectangle represents the projected unit cell, and the dot-dashed lines the $(2 \overline{1} \overline{1} 0)$ projections of the (0001) and (011̄1) planes.

Another mnemonic, perhaps easier to remember than that of Lang (1965), can be visualised in terms of the tetrahedral models of the structures illustrated in figure 3. A careful scrutiny would reveal that Lang's mnemonic is obeyed besides the top edges of the tetrahedra, denoted as $p q$, point outwardly to the leit for $\mathrm{P}_{1} 21$ if we consider the tetrahedra at the top of the distorted hexagonal channels as shown in figure 3 , and to the right for $\mathrm{P}_{2} 21$. It should also be remembered that by considering the edges of the other tetrahedra, a right-

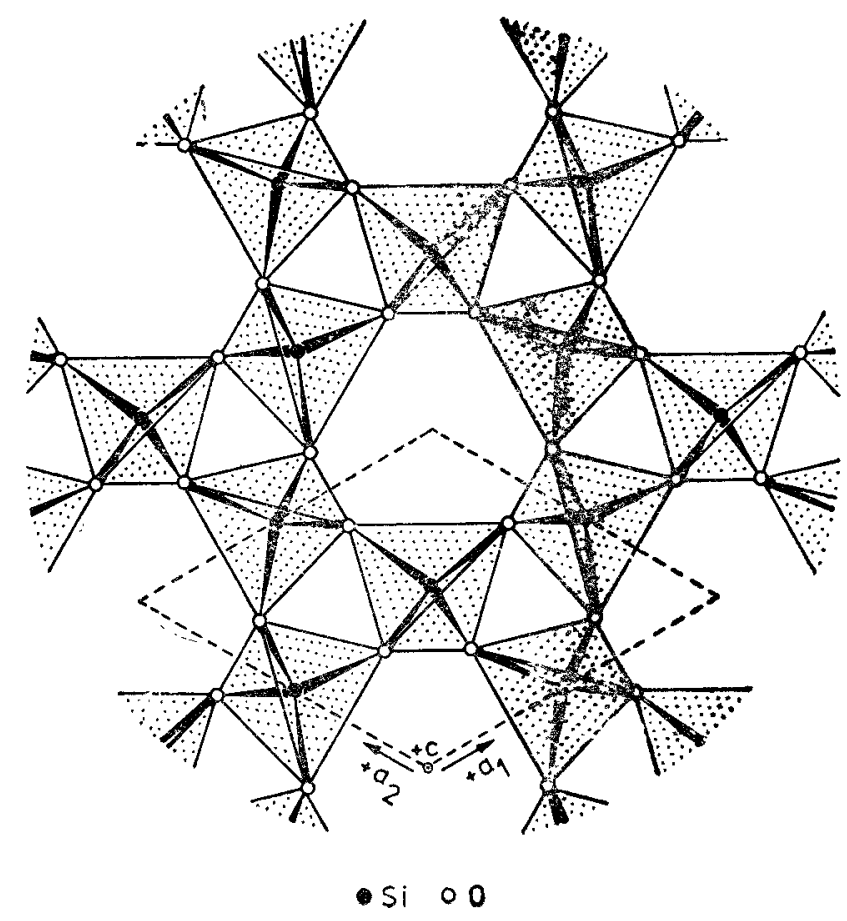

Figure 1a. (0001) projection of the structure of alpha-quartz $\left(\mathrm{P}_{\mathrm{g}} 21\right)$.

* Unless otherwise stated, all subsequent diagrams of alpha-quartz, as sketched in this paper depict the $\mathbf{P 3}_{2} 21$ enantiomorph. 


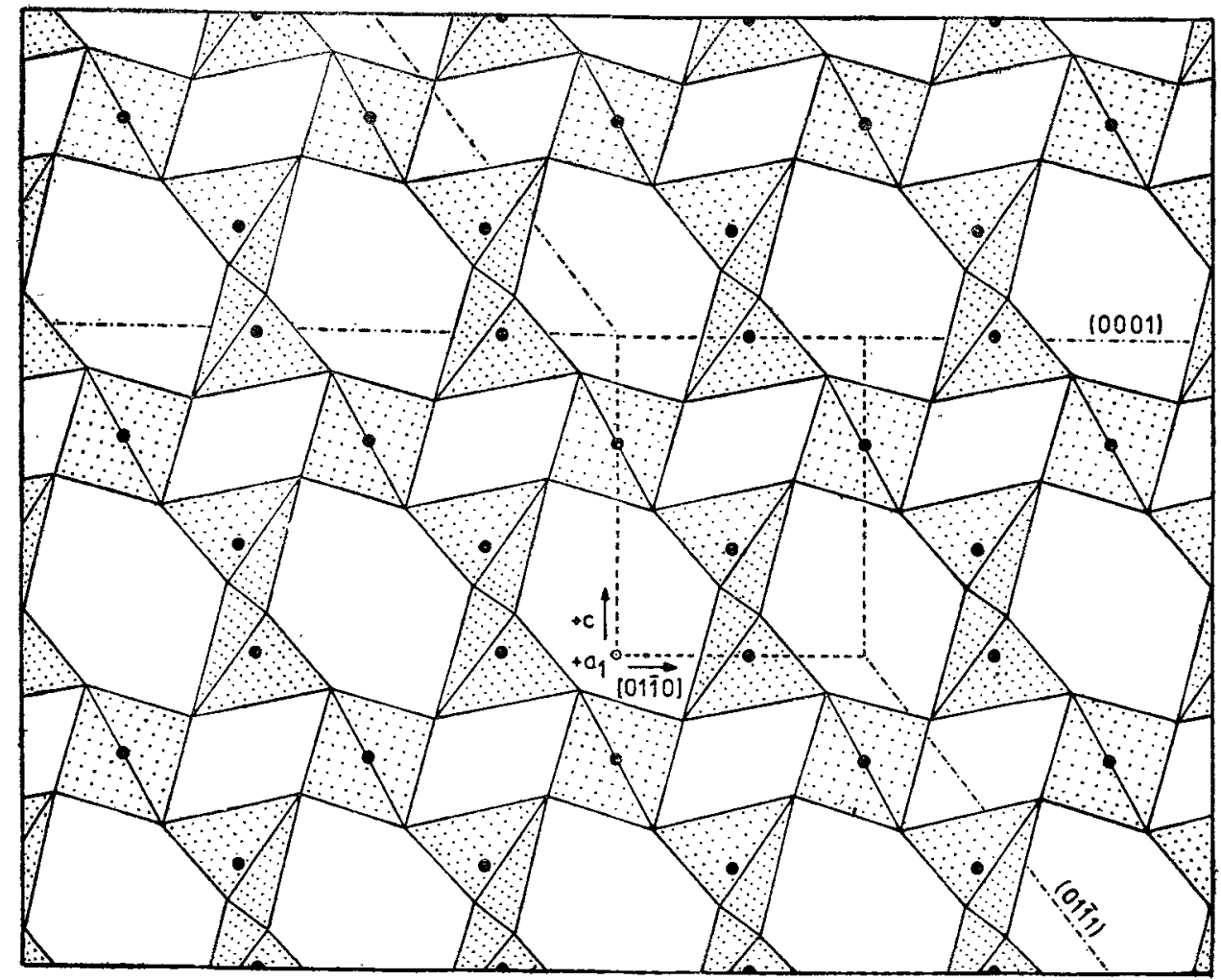

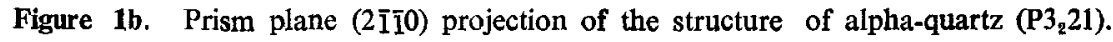

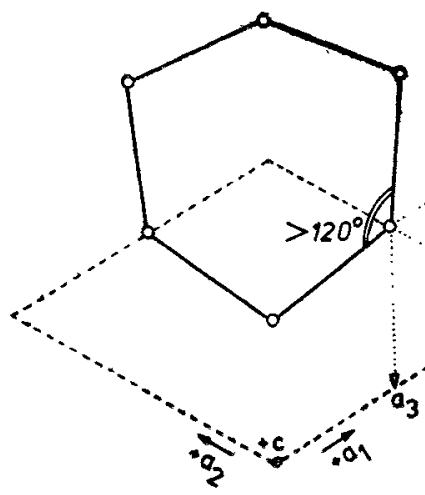

$P 3,21$ (b)

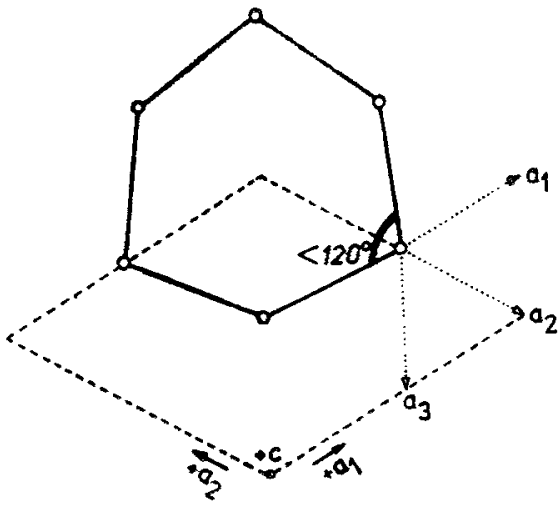

$0 .+$ unit cell
$0 \quad$ si
$\mathrm{P}_{3} 2^{21}$

Figure 2. Diagram illustrating Lang's mnemonic.

handed spiral around the $3_{2}$ axis of the distorted hexagonal channel of $\mathrm{P}_{2} 21$ can be considered (see also figure 1a), as pitch double that of the left-handed spirals (also around $3_{2}$ axes) of the trigonal channels, and vice versa for $\mathbf{P} 3_{1} 21$. 


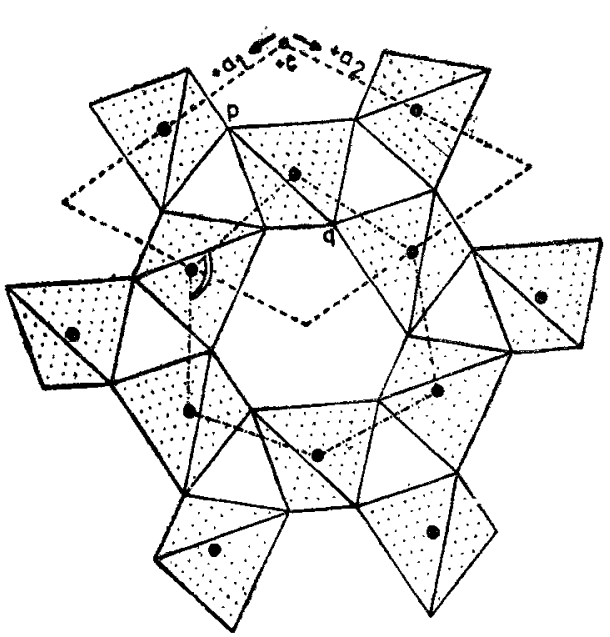

$P 3,21$

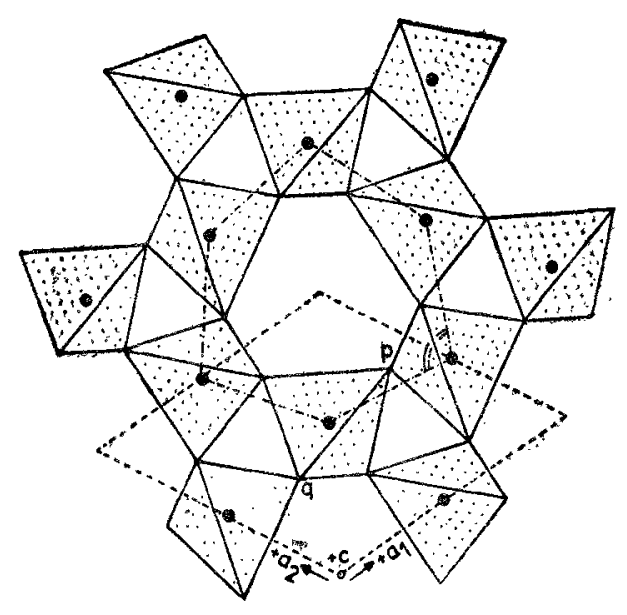

$P 3221$

Figure 3. Mnemonic suggested by the present authors in terms of the tetrahedra models.

Buerger (1956) has defined the $3_{2}$ axis with single pitch (or translation component) as the crystallographically left-handed spiral, and vice versa.

On theoretical considerations, based on optical rotatory power of quartz [Ramachandran (1951), Wooster (1953)] predicted that crystallographically lefthanded alpha-quartz should be morphologically right-handed, as defined by Dana (Ford 1945), and optically dextro-rotatory as well. Wcoster (1953) has defined "optically laevo-rotatory" as "looking towards the source of light the plane of polarisation rotated anticlockwise". Later, De Vries (1958) confirmed Wooster's prediction by determining the absclute configuration of alpha-quartz. All the authors mentioned above used a right-handed axial system, conforming to the International Tables.

Figure 4 illustrates partial stereograms of some of the important planes of the upper halves of crystals of the two enantiomorphic forms of alpha-quartz. The trigonal prism, a $\{11 \overline{2} 0\}$, the trigonal pyramids, $s\{11 \overline{2} 1\}, \mathrm{p}\{11 \overline{2} 3\}$, and the right positive trapezohedron, $\mathrm{x}\{51 \overline{6} 1\}$, are morphologically distinctive features of the $\mathrm{P}_{2} 21$ enantiomorph (Ford 1945); for the $\mathrm{P} 3_{1} 21$ enantiomorph, the distinctive features are the complimentary forms, namely, a $\{2 \overline{1} \overline{1} 0\}$, s $\{2 \overline{1} \bar{j} 1\}$, and $x\{6 \overline{1} 51\}$. The latter forms have also been designated by some authors as 'a $\{2 \overline{1} 10\}$, 's $\{2 \overline{1} 1\}$ and ' $x\{6151\}$ [Bloss and Gibbs (1963)]. The forms a $\{11 \overline{2} 0\}$, a $\{21 \overline{1} 0)$ are rare, and $\mathrm{p}\{11 \overline{2} 3\}, \mathrm{p}\{2 \overline{1} \overline{1} 3\}$, non-existent. The central dots of the stereograms represent (0001), or crystallographically speaking, $(0003)$, since $l=3 n$ for $(000 l)$ type of planes of alpha-quartz, and morphologically speaking, $(000 l)$ is a non-existent system of planes for alpha-quartz.

Twinning is almost universally present in natural quartz. Synthetic quartz grown free from constraints on flawless (including twinning flaws) seed reds or plates do not develop secondary twins (Bandyopadhyay and Saha 1967). Two types of twinning are common in alpha-quartz, namely Dauphine twin and Brazil 

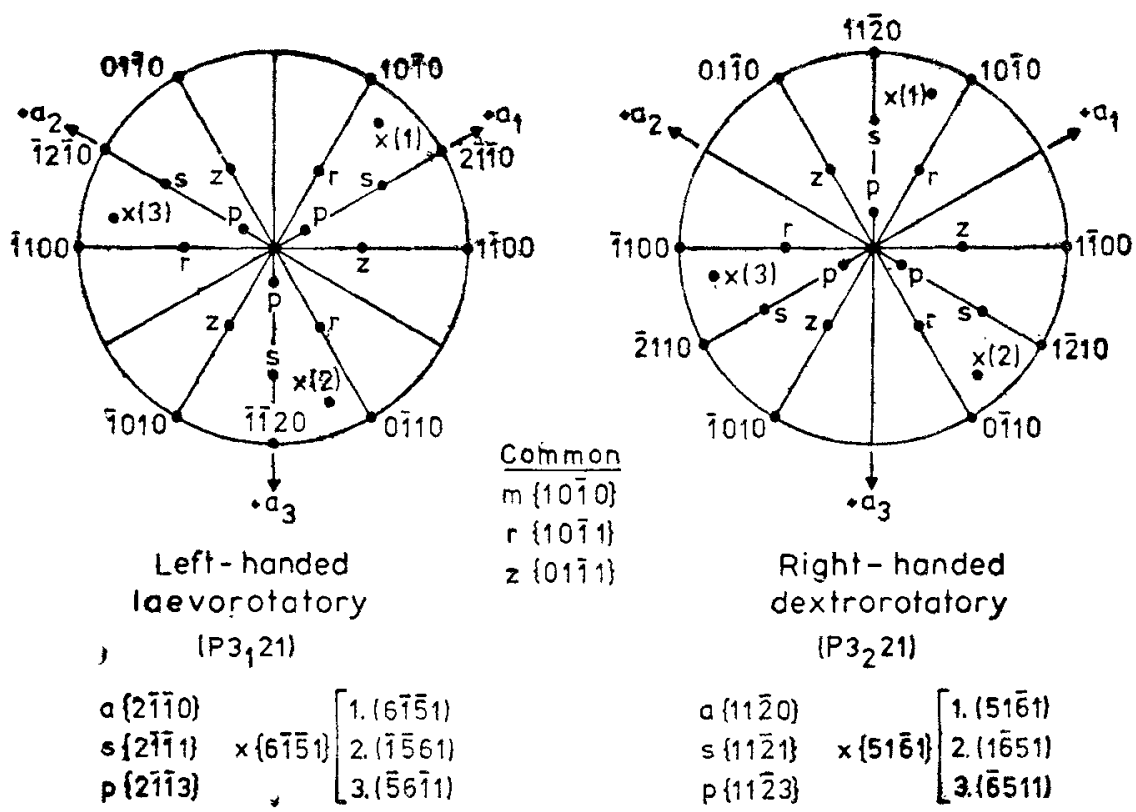

Right-handed

dextrorotatory

$\left(\mathrm{P}_{2}{ }_{2} 21\right)$

$$
\begin{aligned}
& a\{11 \overline{2} 0\} \\
& S\{11 \overline{2} 1\} \\
& P\{11 \overline{2} 3\}
\end{aligned} \quad \times\{51 \bar{\sigma} 1\}\left[\begin{array}{l}
1 .(51 \bar{E} \uparrow\} \\
2 .(1 \overline{\bar{\sigma}} 51) \\
3 .(\overline{6} 511)
\end{array}\right.
$$

Figure 4. Partial stereograms of the enantiomorphs of alpha-quartz.

or optical twin. The resulting forms of Dauphine twin are mostly penetration twins, the twinning-axis being parallel to the $c$-axis, and the twin individuals are both right-handed or left-handed and are asymmetrical. Twinning according to the Brazil law results in irregular interpenetration twins of right- or left-handed individual pairs, the twinning plane being the second order hexagonal prism, a $\{11 \overline{2} 0\}$ (Ford 1945).

\section{Crystal defects}

\subsection{General}

Solids have been broadly divided into two classes on the basis of the ratio of their theoretical shear strength $\tau_{\max }$ and their theoretical cleavage stress $\sigma_{\max }$ (Kelly 1966). Thus, $\tau_{\max } / \sigma_{\max }$ is roughly between $1 / 3$ and 7 for covalently and ionically bound solids, like diamond, sodium chloride, alpha-alumina, eic., and is roughly between $1 / 5$ and $1 / 30$ for metals like alpha-iron (b.c.c.), copper (t.c.c.), etc. (Kelly 1966, tables 1.1 and 1.4). For quartz, it would perhaps be more appropriate to substitute "theoretical fracture stress" for "thecretical cleavage stress", since definition of the word "cleavage" implies to the mineralogist, that the solid separates into two components along a structurally weak plane; quartz does not "cleave" but "fractures" along a curved surface, known as "conchoidal" fracture. A: room temperature, only under very special circumstances can quartz be artificially made to separate along a so-called "planar" surface (Patel et al 1965; Patel 1978). The above analysis, therefore, suggests that a partially ioric crystal like quartz, at room temperature and under uniaxial stress less than its critical fracture stress, is likely to yield by fracturing on microscopic 
scale rather than by slip caused by generation and movement of dislocations. This kind of microfracture formation does not lead to crack propagation and fracturing of the crystals on macroscopic level.

\subsection{X-ray transmission topography}

X-ray transmission topographic studies of as-grown synthetic quartz, have revealed the presence of dislocations (Spencer and Haruta 1966; Lang and Miuscov 1967; McLaren et al 1971; Takagi et al 1974; Auvray and Regreny 1973); further the study by Lang (1967) reveals that the dislocation density in relatively perfect specimens of natural quartz is less by an order of magnitude than that in synthetic quartz.

Figure 5 is a reproduction of the projection topograph of an as-grown synthetic quartz sectioned normal to the $y$-axis (Takagi et al 1974). It may be noted that the dislocations are almost exclusively confined to a column immediately overlying the (0001)-cut surfaces of the seed for the $Z$-zones, fan out for the $-X$ zone from the seed surface, and have an intermediate configuration for the $+X$ zone.

Lang and Miuscov (1967) and McLaren et al (1971) carried out extensive transmission projection topographic studies of as-grown synthetic quartz. Figures $6 a, 6 b, 7$ and 8 are enlarged prints of some projection topographs, and are reproductions of figures $3 a, 3 b, 4 a$ and 5 of McLaren et al (1971), respectively. Table 1 summarises the pertinent data. In contrast to figures $6 a$ and possibly figure 8 , which were taken with prism plane reflections, figure 7 was taken with (0I11) reflections; $c$-axis is therefore vertical in this enlarged print of the topograph in projection only, and hence this print cannot be used to accurately determine inclination of the dislocation lines to the $c$-axis. Figure 8 is an enlarged print of the projection topograph of the final (0001) cobbled surface of crystal X-13; it exactly resembles the optical photomicrograph (of the same area of the same crystal) of figure la of McLaren et al (1971), except that line defects and stacking-fault type of defects, invisible in the photomicrograph, are now in contrast. It can, therefore, be surmised that this topograph was taken with a prism plane type of reflection. Figure 8 is similar to figure 5 of Lang and Miuscov (1967) except that the latter authors used a rhombohedral reflection to take the topograph of part of the final cobbled (0001) surface (possibly a light polish was given to it) of the (0001)-cut plate.

The following general conclusions can be drawn from the studies of Lang and Miuscov (1967) and of McLaren et al (1971):

(i) Dislocations give rise to kinematical, and not dynamical images $(\mu t<1)$.

(ii) Total dislocation density appears to be related to total hydrogen content of the crystals. McLaren et al (1971) estimated that total hydrogen content of the low quality crystals, $X-0$ and $X-13$, is of the order of $5000 \mathrm{H} / 10^{6} \mathrm{Si}$, and their dislocation density about $2.5 \times 10^{3}$ per $\mathrm{cm}^{2}$. The high quality specimen, $\mathrm{Y}-1$, with a hydrogen content too low to be detected, appears to contain much lesser number of dislocations (figure 7 of McLaren et al 1971).

(iii) Z-zone grown portions exhibit growth layering. 
Table 1. Summary of data on x-ray projection topographic studies of McLaren et al. (1971) and Lang and Miuscov (1967) on as-grown synthetic quartz.

\begin{tabular}{|c|c|c|}
\hline & McLaren et al (1971) & Lang and Miuscov (1967) \\
\hline Figure $6 a$ of our paper & Figure $\mathbf{3} a$ & Figure 3 \\
\hline $\begin{array}{l}\text { Specimen } \\
\text { Specimen orientation }\end{array}$ & $\begin{array}{l}X-0 \text {; synthetic** quartz seed } \\
(1 \overline{1} 00) \text {-plate }\end{array}$ & $\begin{array}{l}\text { As-grown synthetic quartz } \\
(1 \overline{2} 10) \text {-plate }\end{array}$ \\
\hline Area studied & Z-zone, including the seed & $\begin{array}{l}\text { Z-zone, not including the } \\
\text { seed }\end{array}$ \\
\hline Topograph* orientation in print & $c$-axis vertical & $c$-axis vertical \\
\hline Reflection used & $11 \overline{2} 0$ & $10 \overline{10}$ \\
\hline g orientation in topograph* & Horizontal & Horizontal \\
\hline Topograph magnification & $10 \times$ & ca. $11 \times$ \\
\hline Dislocation density & $2 \times 10^{3} / \mathrm{cm}^{2}$ & $\begin{array}{l}85 \% \text { of } 3 \times 10^{3} / \mathrm{cm}^{2} \text { that is } \\
\text { ca. } 2.5 \times 10^{8} / \mathrm{cm}^{2} .\end{array}$ \\
\hline Figure $6 b$ of our paper & Figure $3 b$ & Figure 7 \\
\hline Specimen & $X-0$; synthetic** quartz seed & As-grown synthetic quartz \\
\hline Specimen orientation & , &, \\
\hline Area studied & & \\
\hline Topograph orientation in print & $c$-axis vertical & $c$-axis vertical \\
\hline Reflection used & 0003 & 0003 \\
\hline $\mathrm{g}$ orientation in topograph & Vertical & Vertical \\
\hline Topograph magnification & $10 \times$ & ca. $11 \times$ \\
\hline Dislocation density & $5 \times 10^{2} / \mathrm{cm}^{2}$ & $\begin{array}{l}15 \% \text { of } 3 \times 10^{8} / \mathrm{cm}^{2} \text { that is, } \\
\text { ca. } 5 \times 10^{2} / \mathrm{cm}^{2} .\end{array}$ \\
\hline Figure 7 of our paper & Figure $4 a$ & Figure 3 \\
\hline Specimen & $\mathrm{X}-13$; natural ${ }^{* *}$ quartz seed & As-grown synthetic quartz \\
\hline Specimen orientation & (̄1100)-plate & ", \\
\hline Area studied & Z-zone, including the seed & ," \\
\hline Topograph orientation in print & Projection of $c$-axis vertical & ", \\
\hline Reflection used & $0 \overline{1} 11$ & ", \\
\hline g orientation in topograph & Projection horizonta! & ", \\
\hline Topograph magnification & $10 \times$ & ", \\
\hline Figure 8 of our paper & Figure 5 & Figure 3 \\
\hline $\begin{array}{l}\text { Specimen } \\
\text { Specimen orientation }\end{array}$ & & $\begin{array}{l}\text { As-grown synthetic quartz } \\
\text { (0001)-plate }\end{array}$ \\
\hline $\begin{array}{l}\text { Specimen orientation } \\
\text { Area studied }\end{array}$ & $\begin{array}{l}\text { (0001)-plate } \\
\text { Final cobbled (0001) }\end{array}$ & $\begin{array}{l}\text { Part of final cobbled } \\
\text { Pate }\end{array}$ \\
\hline Area studied & surface & (0001) surface \\
\hline Topograph orientation in print & $?$ & Projection of $c$-axis vertical \\
\hline Reflection used & Prism plane (?) & Rhombohedral \\
\hline$g$ orientation in topograph & & Projection vertical \\
\hline Topograph magnification & $10 \times$ & ca. $11 \times$ \\
\hline
\end{tabular}

* Topograph in this context signifies enlarged prints of the topographs as shown in the paper of McLaren et al 1971 and reproduced here, and in the paper of Lang and Miuscov (1967).

** For figures $6(a)$ and (b) of our paper, McLaren et al (1971) suggested that the seed was derived from synthetic quartz. They were more definite about the source of the seed (natural quartz) of figure 7; see, McLaren and Retchford (1969). 


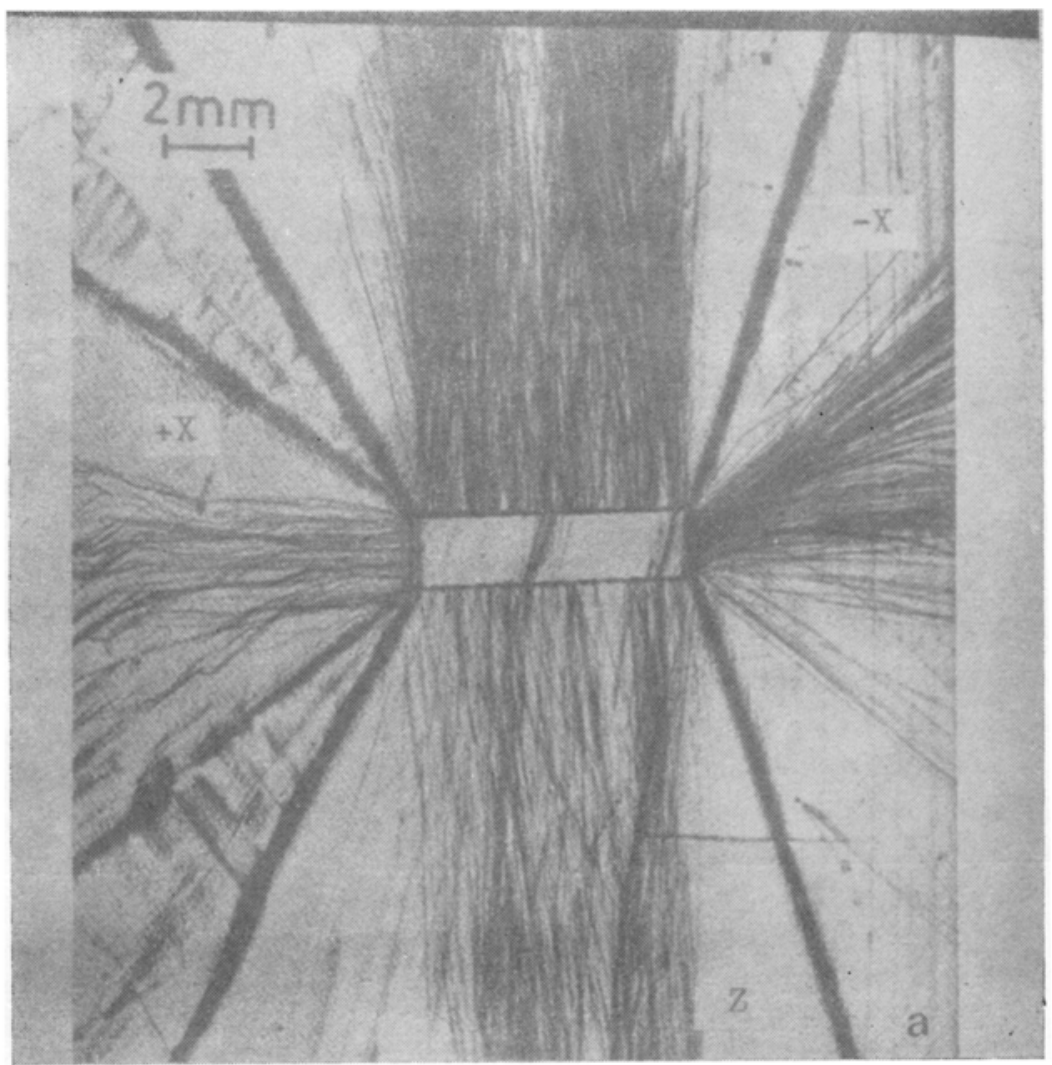

Figure 5. $2 \overline{1} \overline{1} 0$ projection topograph of a $Y$-cut plate of synthetic quartz showing growth sectors $+X,-X, s$ and $Z$, and the sector areas bounded by highly strained boundaries. Radiation : $\operatorname{MoK}_{\alpha}$ (Courtesy: Dr. M. Takagi). 


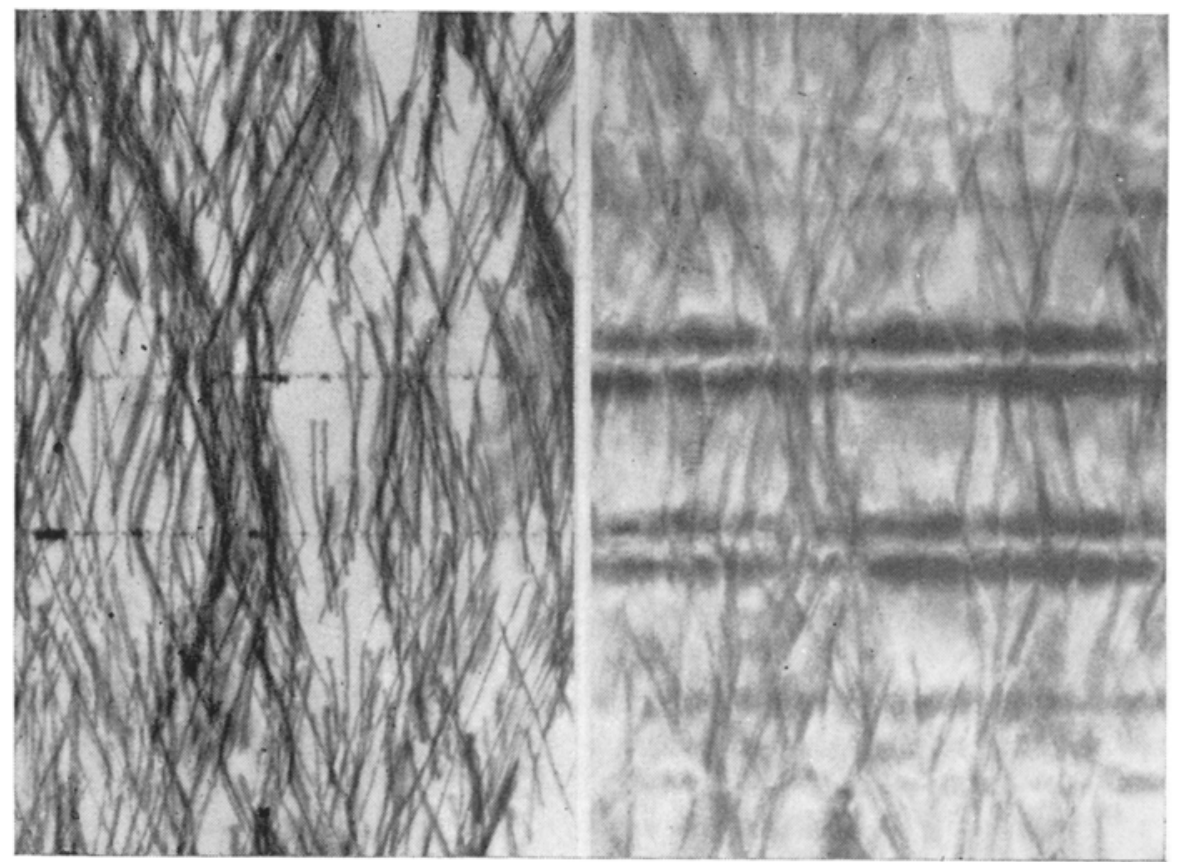

(a)

(b)

Figure 6a. $11 \overline{2} 0$ projection topograph of a $m(\overline{1} 100)$ plate of synthetic crystal $X-0$. showing the synthetic seed area and bundles of dislocations continuing into the grown portions. Radiation: $\mathrm{AgK}_{a}$. Magnification : $10 \times$.

Figure 6b. 0003 projection topograph of a $m$ (i100) plate of synthetic crystal $\mathrm{X}-0$, showing the high contrast seed-crystal interface. Radiation: $\mathrm{AgK}_{\alpha}$. Magnification : $10: \because$. 


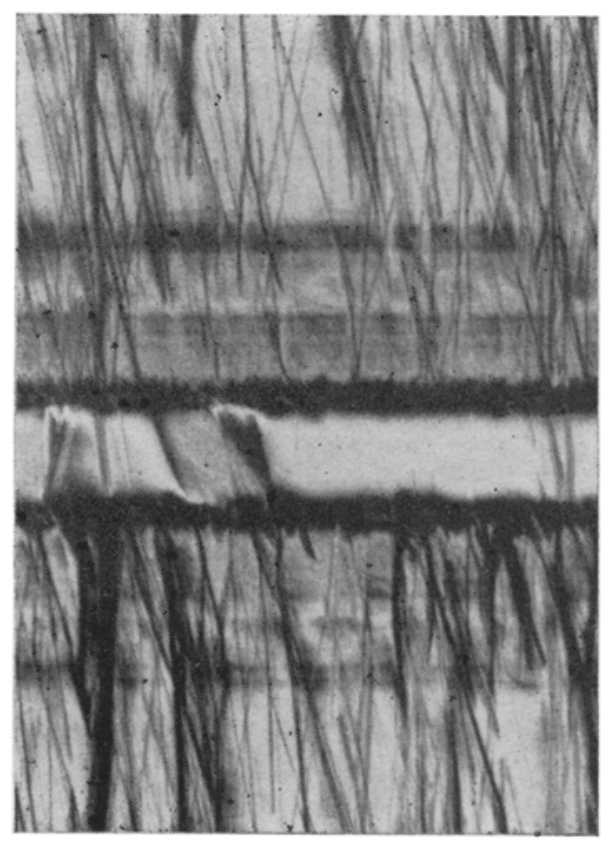

Figure 7. $0 \overline{1} 11$ projection topograph of a $\mathrm{m}(\overline{\mathrm{l}} 100)$ plate of synthetic crystal $X-13$ showing high contrast seed-crystal interface with natural seed area almost free from dislocations, and grown portions with dislocations generated at the seed surface. Radiation : $\mathrm{AgK}_{\boldsymbol{a}}$. Magnification : $10 \times$. 


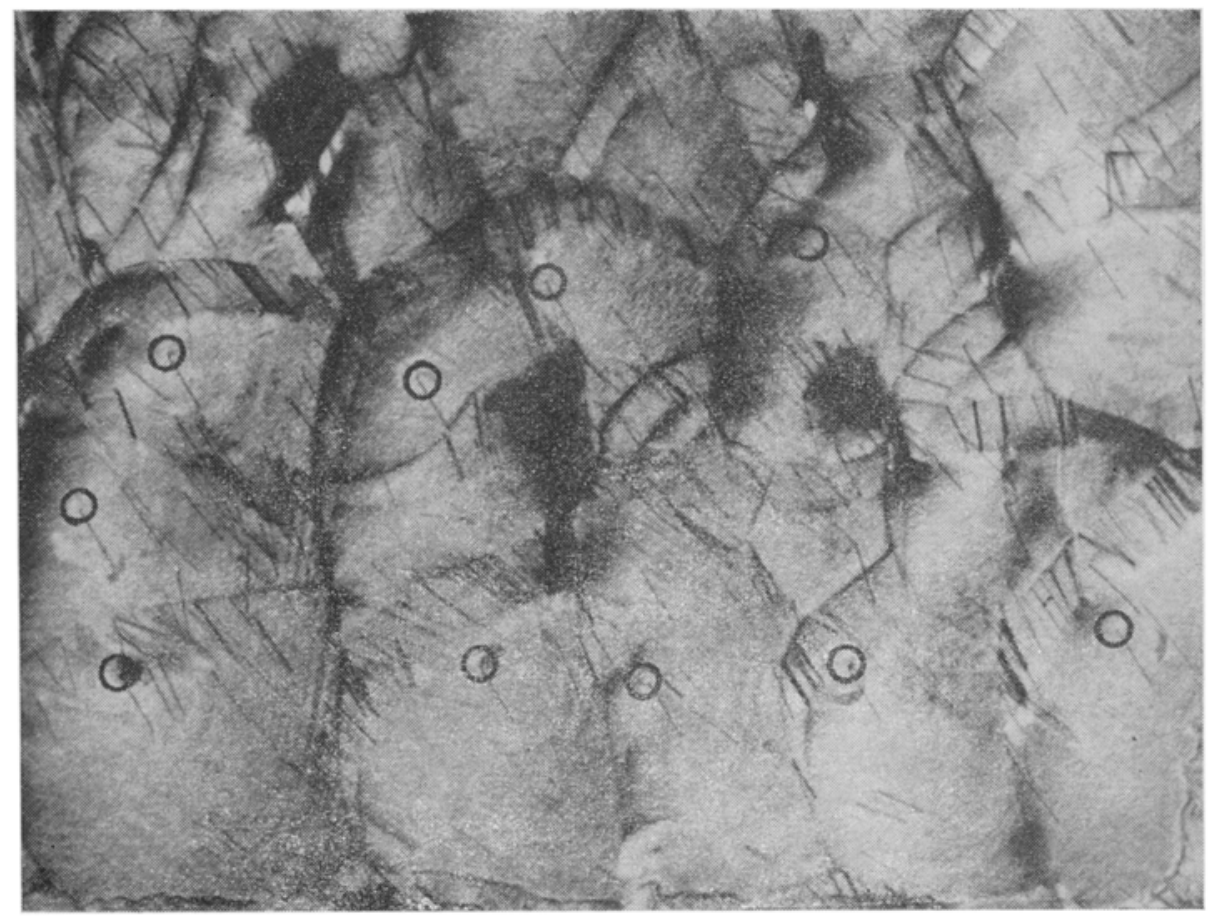

Figure 8. "Prism plane" projection topograph of a (0001) slice of synthetic crystal $\mathrm{X}-13$, showing $1: 1$ correspondence of a few dislocations (circled) with the apices of most of the cobbles, and others confined to the cell boundaries. Radiation : $\mathrm{AgK}_{a}$. Magnification: 10 . (Figures 6-8, Courtesy: Dr. A. C. McLaren). 
(iv) Natural quartz seed is relatively free from dislocations (figure 5 of this paper reproduced from. Takagi et al 1974) and shows a relatively broad band of intense contrast at the interfaces between the seed and grown portions (figure 7 of this paper). The few dislocations that traverse the seed are also propagated in the grown portions where it is much greater. This suggests that dislocations are generated at the seed surfaces and grow with the synthetic crystal (McLaren et al 1971). The synthetic quartz seed, on the other hand, shows no intense contrast at the interfaces in the topograph taken with $\{1120\}$ reflection (figure 6a), but does so in the topograph taken with (0003) reflection (figure 6b). Furthermore, there is almost $1: 1$ correspondence between the dislocations in the seed and in the grown portion.

(v) Images are single for topographs taken with high intensity reflections, like $\{10 \overline{1} 1\}$, but double for low intensity reflection like (0003), and for $\{11 \overline{2} 0\}$ under special circumstances (figures 8a and 8c of McLaren et al 1971). This corresponds with theory (Tanner 1976).

(vi) Dislocation lines are relatively straight, but are not parallel to the $c$-axis; they are actually confined to a cone of about $25^{\circ}$ around the $c$-axis (figures $6 \mathrm{a}, 6 \mathrm{~b}$ and figure 5 of this paper).

(vii) Stacking-fault defects are not so clear in figure 8 of this paper as it is in figure 5 of Lang and Miuscov (1967); however, their presence in figure 8 helped to identify the final (0001) cobbled surfaces of synthetic quartz as surface manifestations of the cell structure in the Z-zones of the synthetic crystals. From figure 8 , it appears that two sets of the high-curvature fault surfaces at the cell boundaries, in approximate parallel alignments, are in contrast, possibly controlled by the orientation of the specific prism plane reflection used for taking the topograph. From figure 5 of Lang and Miuscov, it also becomes clear that must of the dislocations are confined to fault fringes at the cell walls (those have been designated in this paper as "cell wall dislocations").

(viii) Fault fringes at cell walls have a fault displacement vector of about $1 \AA$ associated with them. The imperfect thin layers at the cell walls are a few microns thick, and are probably caused by segregation of a substantial fraction of the impurities at the cell walls (Lang and Miuscov 1967).

(ix) Figure 8 also indicates that only a single dislocation, localised in the figure with the help of open circles, is assciated with the distinctive apex of each type II hillock or cobble (figure 1a of McLaren et al 1971; Bandyopadhyay and Saha 1966) of the terminal (0001) surfaces of crystal X-13. Those have been designated in this paper as "cobble-apex dislocations". Their number is small compared to the cell-wall dislocations.

Lang and Miuscov (1967) and McLaren et al (1971) have also attempted to determine the Burgers vector of dislocations in synthetic quartz using the invisibility rule. Their conclusions can be examined in the light of the conditions for images of strain fields of dislocations guing out of contrast in the topographs.

Invisibility rule: Conditions for disappearance of the image of the strain field of a dislocation in x-ray diffraction topographs are given in figure 9. For a 
pure screw dislocation, since the Burgers vector (b) is parallel to the dislocation line $\left(I_{s}\right)$, the diffraction vector $(g)$ must be normal to both the Burgers vector and the dislocation line for the image to go out of contrast, or in other words,

$$
\mathbf{g} \cdot \mathbf{b}=0
$$

For a pure edge dislocation $\left(\mathbf{l}_{\sigma}^{\prime}\right.$; not shown in the figure) and a mixed dislocation $\left(\mathfrak{l}_{m}^{\prime}\right)$ in a plane containing the diffraction vector $\left(\mathrm{g}^{\prime}\right)$ and the Burgers vector (b),

$$
\mathbf{g}^{\prime} \cdot \mathbf{b} \times \mathbf{l}=\mathbf{0}
$$

for disappearance of the image.

If the reflection is so chosen that the $\mathrm{g}$ coincides with the line of the pure edge dislocation ( $I_{0}$ in figure 9 ; this is a special case), then condition (1) is satisfied in addition to condition (2), and the image goes out of contrast.

On the other hand, if $\mathbf{l}_{c}^{\prime \prime}$ does not coincide with the plane containing $\mathbf{g}$ and $\mathbf{b}$ then it will show contrast. The contrast will be maximum when $l_{0}^{\prime}$ is normal to this plane ( $l_{e}^{\prime \prime}$ has not been shown in the figure, to avoid confusion).

A general mixed dislocation $\left(\mathbf{l}_{\boldsymbol{m}}\right)$ can never be invisible. However, the image is likely to become faint, if the line of the dislocation makes a small angle $\left(90^{\circ}-\gamma\right.$; figure 9) with the plane containing $\mathbf{g}^{\prime}$ and $\mathbf{b}$.

Approximately $80-85 \%$ of the dislocation lines of figure 6 a taken on a (I100) slice with a prism plane reflection, go out of contrast in figure $6 \mathrm{~b}$, taken with $(0003)$; from this observation it was concluded that they were cell-wall dislocations and that the Burgers vector of those dislocations would be confined to the (0001) plane. This would imply that the dislocations should be pure edge dislocations with the line of the dislocations strictly parallel to the $c$-axis $\left(l_{\theta} ;\right.$ see figures 9 and 10). However, from figure $6 \mathrm{a}$ it can be seen that this is not so. The

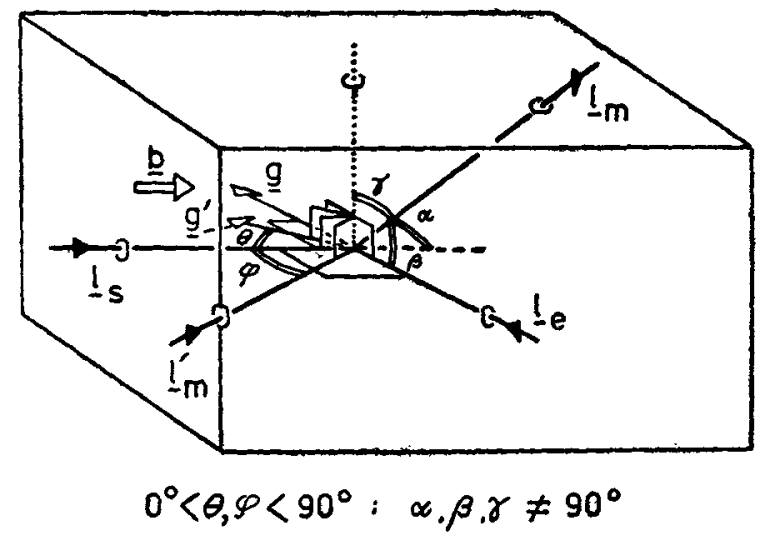
(a) Pure screw $\left(\underline{l}_{s}\right)$ :
$\underline{g}, \underline{b}=0$
(b) Pure edge (!e)
(c) Special mixed $\left(\underline{I}_{m}^{\prime}\right)$ ]
$\underline{g^{\prime}} \cdot \underline{b} \times \underline{l}=0$
(d) General mixed ( $\left.I_{m}\right)$ : never invisible

Figure 9. Diagrammatic sketch illustrating the relations between diffraction vectors, lines of the dislocations and their Burgers vector. 


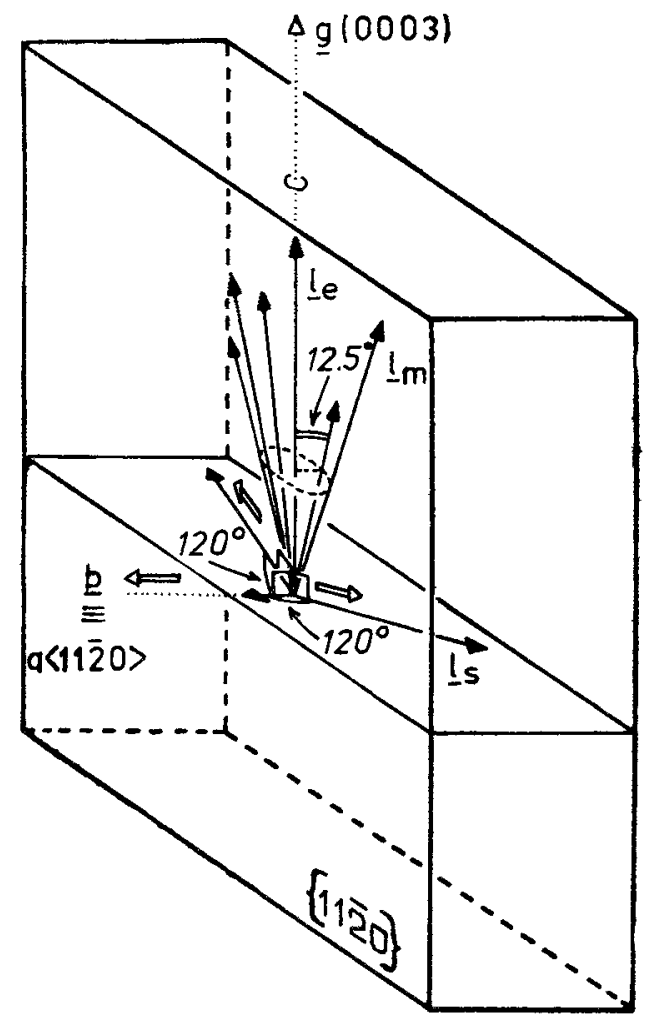

Figure 10. Diagrammatic sketch illustrating the alignments of a-type cell-wall dislocations $\left(\mathrm{I}_{\theta}\right)$ confined within a cone of $25^{\circ}$ around $c$-axis, as found in synthetic quartz.

authors, therefore, suggested that the cell-wall dislocations were of a mixed nature, with a strong edge component; the slip planes characterising the strong edge cumponent of the cell-wall dislocations wotild therefore, be of the prism plane type. As a corollary, we can conclude that mixed cell-wall dislocations cannot normally go completely out of contrast and that their disappearance in figure $6 \mathrm{~b}$ can be attributed to the small angles they make with the $c$-axis which is also the direction of the diffraction vector in this case (Tanner 1976).

McLaren et al (1971) further extended this study in order to define the exact nature of the basal celi-wall dislocations. A (0001) slice of crystal Y-1, with a low density of dislocations, was selected for this purpose. Investigating eleven specific cell-wall dislocations as well as two clusters with the six rhombohedral $\{10 \overline{1} 1\}$ reflections, they came to the conclusion that the dislocations are of the type $\mathbf{b}=a\langle 11 \overline{2} 0\rangle$, i.e., they are a-type dislocations. If these be a-type pure edge dislocations, the alternative to figure 10 would be as illustrated in figure 11. In a (1100) slice the dispositions of the three groups, $\mathbf{I}_{\theta_{1}}, \mathbf{I}_{\theta_{2}}$ and $\mathbf{I}_{e_{3}}$, of a-type pure edge dislocations would be as shown in the figure. If a (1101) reflection is used, $I_{a_{1}}$, characterised by a Burgers vector $b_{1}=a[11 \overline{2} 0]$ and a slip plane containing $\mathbf{b}_{1}$ and $\mathbf{g}$ should go out of contrast, since it satisfies both conditions (1) and (2) 


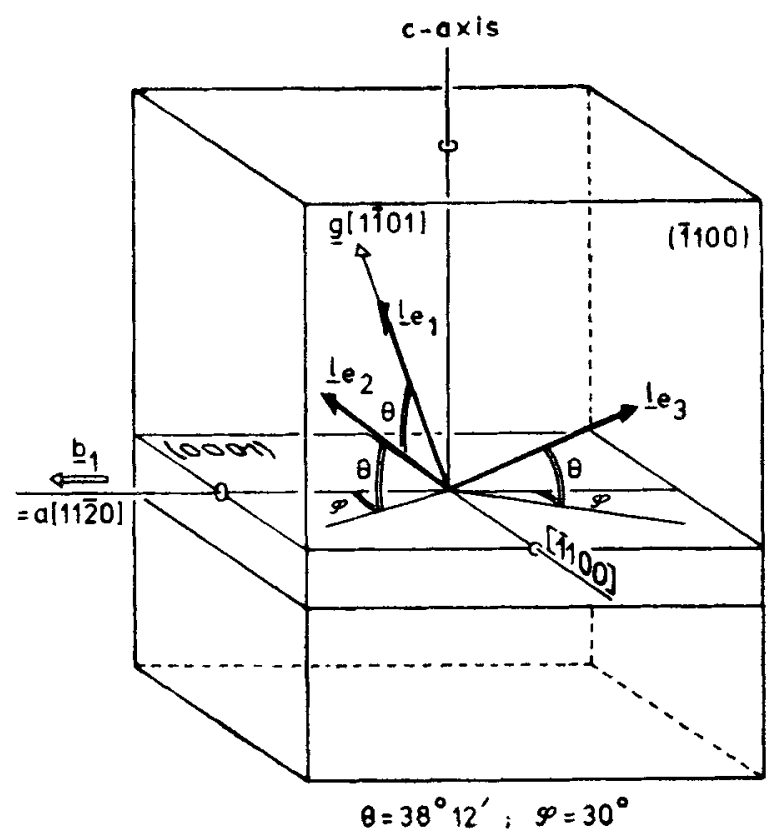

Figure 11. Diagrammatic sketch illustrating a hypothetical alternative configuration of the a-type cell-wall dislocations.

mentioned above (figure 9), but $\mathbf{l}_{\boldsymbol{e}_{2}}$ and $\boldsymbol{t}_{\boldsymbol{\theta}_{\mathrm{a}}}$ should remain in relatively strong contrast making a large angle (ca. $55^{\circ}$ ) with the $c$-axis, since the interfacial angle $\theta$ between the corresponding crystallographic planes of the two groups, $\{1100\}$ and $\{\overline{1} 101\}$, is $38^{\circ} 12^{\prime}$ (figure 11 ; Frondel 1962). This is contrary to the alignment of dislocations in figure 6 a taken on a $(\overline{1} 100)$ slice (though with a 1120 reflection), specially since this topograph should give an undistorted view of alignments of the projected images of cell-wall dislocations. On the other hand, if the cell-wall dislocations are imagined to be a-type mixed dislocations $\left(l_{n}\right)$ they should never have gone out of contrast when $\{\overline{1} 101\}$ reflections were used to take the topographs on a (0001) slice (as was done by McLaren et al 1971), since the angle between the dislocation lines and the corresponding planes containing $\mathbf{g}$ and $b$ should then have been $42^{\circ}$ or more (after making allowance of about $12^{\circ}$ for deviation of the lines of the dislocation images (figure 6a) frum the $c$-axis), and condition (2) given above (figure 9) would never have been satisfied.

In order to explain this anomaly, it can be postulated that a-type cell-wall dislocations may be constituted of two alternating components, i.e., a pure edge a $\langle 1120\rangle$-type conservative glide component parallel to $c$-axis operating on the corresponding $\{10 \overline{1} 0\}$ planes, and a non-conservative climb component making an angle of ca. $52^{\circ}$ with the $c$-axis, with the glide component by far predominating over the climb component. This can perhaps explain (1) the disappearance of all the cell-wall dislocation images in a (1100) slice when a (0003) reflection is used (figure 6b), because of pieponderance of the glide component, (2) disappear- 
ance of particular sets of images in a (0001) slice when complimentary pairs of high intensity $\{1 \overline{1} 01\}$ reflections are used, since the preduminating glide components will be reduced to point images in (0001) projection, and (3) the cone of $25^{\circ}$ around the $c$-axis in which the dislocation lines are cortained (figures $6 \mathrm{a}$ and 10). It may be pointed out that a topograph of (0001) sice, and to a lesser extent that of a $\{10 \overline{1} 1\}$ slice (figures 4 and 5 of Lang and Miuscov 1967) show very regular alternate broadening and thinning out of the images of cell-wall dislocations. This feature, however, is not evident in the work of McLaren et al (1971) on a (0001) slice of crystal $X-13$. The difficulties encountered in our attempts to determine the Burgers vector of the cobble-apex dislocations, which constitute the rest 15$20 \%$ of the dislocations in synthetic quartz, are somewhat similar in nature. Strong contrast double images of those dislocations are obtained in the topographs taken with 0003 reflection (figure 6b), but it is doubtful whether they go completely out of contrast in the topographs taken with $11 \overline{2} 0$ (figure 6a), or with $10 \overline{1} 0$ reflection (figure 3 of Lang and Miuscov 1967). Moreover, rarely are they parallel to the $c$-axis in the topographs taken with 0003 reflection. These observations preclude the majority of them from being pure c.type screw dislocations Using all six rhombohedral reflections $\{10 \overline{1} 1\}$ on a (0001) plate of crystal X 13, McLaren et al. (1971) attempted to determine the Burgers vector of the cobble apex dislocations (figure 8). Their conclusion was that those dislocations may be of mixed character with $\mathbf{b}=:(a+c)[1 \overline{2} 13]$. However, no evidence of the other two directions of the set, $\mathbf{b}=(a+c)\langle 1 \overline{2} 13\rangle$, was found, though alignments of the cobble-apex dislocations in topographs of figure $6 \mathrm{~b}$, and of figure 7 of Larg and Miuscov (1967), suggest that they should have been present. Moreover, energetically it would further seem to be unlikely that dislocations with two types of Burgers vectcr $\mathbf{b}=c$ [0001] and $\mathbf{b}=(a+c)$ [1213] which ar, scmewhat parallel to each other (see figure 4) should give rise to the same type (cobble-apex) of screw dislocations in synthetic quartz.

Auvray and Regreny (1973) investigated as-grown high quality synthetic crystals using both projection transmission topography and infrared technique. These studies provide the following information:

(i) Boundaries of the seed are practically invisible in the topugraph (figure 1 of Auvray and Regreny 1973) taken with $2 \overline{1} 10$ reflection (cf. figure 6a). However, it must be pointed out that very intense double image contrast of the (0001) seed-crystal interfaces is brought out in the topograph of the same crystal (X-0) taken with 0003 reflection (figure 6b). Also, the topograph of crystal X-13 taken with $0 \overline{1} 11$ reflection shows strong contrast at the (0001) seed-crystal interfaces (figure 7). Since Auvray and Regreny (1973) always usud $2 \overline{1} 0$ reflection, it appears that absence of contrast at (0001) seed crystal interfaces is therefore no proof of absence of strain at the same places.

(ii) The region in the Z-zone overlying a relatively dislocation-free portion of the synthetic seed (marked $l$ in their figure 1) not only contains less dislocations than the region in the Z-zone overlying a portion of the same seed containing more dislocations (marked $\mathrm{H}$ in their figure 1), but also the mechanical Q of the portion I is greater by $15 \%$ than that of portion $\mathbf{H}$. This is perhaps the first time that the density of dislocations in synthetic quartz has been correlated with variation of acoustic loss of the crystal 
in different portions. Since $85^{\circ}{ }_{0}$ of the dislocations of synthetic quartz have been characterised as a-typc "cell-wall dislocations", it would perhaps not be wrong to conclude that they contritute significantly to lowering of mechanical Q of synthetic quartz crystals.

\section{Conclusions}

A critical analytical study of the crystallography of alpha-quartz and the nature of crystal defects found in synthetic quartz (i.e., crientation of dislocations, their localisation. etc.) has been made on the basis of previcus $x$-ray diffraction topographic studies and application of the invisibility rule to the data.

A mnemonic based on the tetrahedral model representation of the alphaquartz structure, perhaps easjer to remember than that of Lang 1965, has beets suggested fur characterisation of the enantiomorphs in conformity with modern international practice (International Tables).

A brief reference to the strength of a patrially ionic maicrial like alpha-quartz has been made. and the type of mechanism likely to influence its failure behaviour under stress has beer indicated. Since the prinary requisite for dislocation movement in crystalline material is the presence of a slip system, which is absent in alpha-quartz at rocm temperaturc and pressure, a fraciure mechanism active on a submicroscopic scale has been suggested.

Direct images of the strain fields of dislocations in as-grown undeformed synthetic quartz were obtained among others by Lang and Miuscov (1967), McLaren et al (1971), Auvray and Regreny (1973) and Takagi et al (1974). Some of the important observations of these workers are :

(i) Good specimens of natural quartz single crystals have fewer dislocations as compared to the synthetic ones.

(ii) Dislocation densities of synthetic quartz are related to their hydrogen impurity content; a higher dislocation density not only signifies higher concentration of hydrogen impurity, but a lower mechanical $Q$ as well. Density estimated in low quality synthetic quartz with high hydrogen impurity content (ca. $5000 \mathrm{H} / 10^{6} \mathrm{Si}$ ) is of the order of $2.5 \times 10^{3}$ per $\mathrm{cm}^{2}$ (McLaren et al 1971), whereas the same is much less $\left(<10-10^{2}\right.$ per $\left.\mathrm{cm}^{2}\right)$ in high quality crystals (Auvray and Regreny 1973).

(iii) All dislocations, whether present in the seed crystals or generated at the seed-crystal interfaces and within the grown porions, are propagated in the direction of growth.

(iv) Normally, dislocations present in the Z-zones are contained within a column overlying the (0001)-cut seed surfaces.

(v) About $80-85 \%$ of the dislocations in synthetic quartz are pure edge dislocations confined to a cone around the $c$-axis making an angle of about $25^{\circ}$, with $\mathbf{b}=a\langle 1120\rangle$. These dislocations are distributed along the cell boundaries giving them the appearance of stacking fault-type boundaries with a displacement vector of about $1 \AA$. The cell boundaries consist of an imperfect thin layer a few microns thick, being a region of considerable impurity segregation. These have been designated by the present authors as cell-wall dislocations. 
(vi) The rest $10-15 \%$ dislocations are predominantly screw dislocations with a large c-component, and each of them is associated with a single cobble apex (type II). Those dislocations have been designated by the present authors as cobble-apex dislocations.

Finally, a critical analysis of the x-ray diffraction projection topographic images of the dislocations in synthetic quartz has been carried out by the present authors employing primarily the invisibility criterion. Suggestions have been made to explain the earlier anomalies.

(i) $80-85 \%$ of the dislocations present in the cell boundaries in synthetic quartz may be a-type dislocations with a pure edge a $\langle 11 \overline{2} 0\rangle$ glide component parallel to $c$-axis and operating on $\{10 \overline{1} 0\}$ planes, ard an alternating climb component making a large angle with the $c$-axis, resulting in an overall configuration of the dislocations which can best be described by the statement, "the lines of the cell-wall dislocations are confined to a cone of $25^{\circ}$ around the $c$-axis". This would suggest that the glide component by far predominates over the climb component.

(ii) The real nature of the cobble-apex 'screw' dislocations is not understood from the present analysis, but a mechanism similar to (1) above may be postulated to explain deviation of the dislocation lines from strict parallelism with the $c$-axis. However, further high resolution x-ray diffraction topographic studies are necessary.

\section{Acknowledgements}

The authors are indebted to the Director, CGCRI, for his kind permission to publish this paper. Thanks are also due to $\mathrm{Dr} M$ Takagi and Dr A C McLaren for their kind permission to reproduce some of the $\mathrm{x}$-ray transmission projection topographs from their papers.

\section{References}

Anon 1969 International tables for $x$-ray crystallography (Birmingham: The Kynoch Press) Vol. 1 Auvray P and Regreny A 1973 Bull. Soc. Fr. Mineral. Crystallogr. 96267

Bandyopadhyay T and Saha P 1966 Cent. Glass Ceram. Res. Inst. Bull. 1359

Bandyopadhyay T and Saha P 1967 Cent. Glass Ceram. Res. Inst. Bull. 14105

Bloss F D and Gibbs G V 1963 Am. Min. 48821

Buerger M J 1956 Elementary crystallography (New York : John Wiley)

De Vries A 1958 Nature (London) 1811192

Ford W E 1945 A textbook of mineralogy (New York: John Wiley)

Frondel C 1962 The system of mineralogy Vol. 3-Silica Minerals (New York: John Wiley)

Kelly A 1966 Strong solids (Oxford : Clarendon Press) p. 212

Kingery W D, Bowen H K and Uhlmann D R 1976 Introduction to ceramics 2nd ed. (New York : John Wiley)

Lang A R 1965 Acta Crystallogr. 19220

Lang A R 1967 Proc. Int. Conf. on Crystal Growth, Boston, U.S.A., ed. H. S Peiser, (Oxford : Pergamon Press)

Lang A R and Miuscov V F 1967 J. Appl. Phys. 382477

McLaren A C and Retchford J A 1969 Phys. Status solidi 33657

McLaren A C, Osborne C F and Saunders L A 1971 Phys. Status solidi A4 235 
Muan A and Osborn E F 1965 Phase equilibrium among oxides in steel making (Massachusetts: Addison-Wesley) p. 178

Patel A R 1978 Personal Communication

Patel A R, Bahl O P and Vagh A S 1965 Acta Crystallogr. 19757

Ramachandran G N 1951 Proc. Indian Acad. Sci. A34 127

Spencer W J and Haruta K 1966 J. Appl. Phys. 37549

Takagi M, Mineo H and Sato M 1974 J. Cryst. Growth 24/25 541

Tanner B 1976 X-ray diffraction topography (Oxford : Pergamon Press) p. 37

Wooster W A 1953 Rep. Progr. Phys. 1662

Wyckoff R W G 1931 The structure of crystals 2 edn. (New York: Chemical Catalog) p. 242

Wyckoff R W G 1948 Crystal structures (New York : Interscience Publishers) $§ 1$ 